\title{
Forearmed and deceived: Diagnosis?
}

\author{
LJ Miedzinski $\mathrm{MD}^{1}$, J Mahood $\mathrm{MD}^{2}, \mathrm{M} \operatorname{Sin} \mathrm{MB} \mathrm{BS}^{3}, \mathrm{G}$ Sterling $\mathrm{MD}^{3}$
}

A 58-year-old male with a four-year history of noninsulin dependent diabetes presented in early January 1999 with a two-week history of progressive right forearm swelling, pain, and limitation of wrist and elbow movement unassociated with recognized trauma. $\mathrm{He}$ had been febrile in the three days before the evaluation, despite having been on an anti-inflammatory medication for approximately one week. Progressive clinical worsening in association with a markedly elevated erythrocyte sedimentation rate prompted referral to an infectious diseases consultant regarding the possibility of osteomyelitis.

On examination, the patient was flushed, tachycardic at 110 beats $/ \mathrm{min}$ and mildly febrile, with a temperature of $38.2^{\circ} \mathrm{C}$. Blood pressure was $150 / 90 \mathrm{mmHg}$ in the right arm sitting. Physical examination revealed no conjunctival petechiae, normal heart sounds and specifically no cardiac murmurs. The most relevant physical abnormality was an indurated right forearm, edematous from the elbow to the metacarpophalangeal joints of the hand. There was minimal erythema over the dorsum of the hand and wrist, and no deep tenderness to palpation of the muscles. Although there was no limitation to passive movements of either wrist or elbow, there was marked limitation of active wrist and elbow extension.

White blood count was elevated at $17.0 \times 10^{9} / \mathrm{L}$, with a normal hemoglobin of $142 \mathrm{~g} / \mathrm{L}$ and platelet count of $295 \times 10^{9} / \mathrm{L}$. Creatinine was normal at $77 \mu \mathrm{mol} / \mathrm{L}$ with a normal creatine kinase of $58 \mathrm{U} / \mathrm{L}$. The random glucose level was elevated to $16.5 \mathrm{mmol} / \mathrm{L}$. An x-ray of the arm showed no gas in the soft tissues nor any changes suggestive of osteomyelitis.

Because of the possibility of group A streptococcal fasciitis, an orthopedic evaluation was requested. The surgeon felt confident that the patient did not have compartment syndrome nor did he think invasive fasciitis was likely. Blood cultures were obtained, and the patient was started on empirical intravenous clindamycin and ceftriaxone.
By the next day, the patient's blood cultures were reported positive for Staphylococcus aureus, resistant to penicillin but sensitive to oxacillin. A bone scan showed diffuse hyperemia of the forearm "consistent with an inflammatory condition such as cellulitis" and no evidence of osteomyelitis. The patient's antibiotics were changed to intravenous cloxacillin. Over the next three days, the patient showed little change in the appearance or discomfort of his arm despite a decreasing fever and decreasing leukocytosis.

A diagnostic procedure was performed. What is your diagnosis?

\section{DIAGNOSIS}

A computerized axial tomogram (CT) scan of the arm was obtained on the sixth day of hospitalization (Figure 1). It shows thickening of the subcutaneous tissue with an extensive multiloculated fluid collection extending from the level of the wrist to the elbow, anteriorly and posteriorly through the interosseous space. Adjacent muscles appeared swollen. The radiologist's interpretation was that it was markedly abnormal with changes "in keeping with diffuse cellulitis, abscess formation and possible myositis". An ultrasonographic examination revealed "a hypoechoic volar compartment with loss of normal muscle striation”. The sonographically abnormal area was aspirated and a drain was inserted, releasing $40 \mathrm{~mL}$ of purulent material, which on Gram stain showed 4+ polymorphonuclear leukocytes and Gram-positive cocci in clusters. The aspirate yielded growth of $S$ aureus with the same sensitivity pattern as the blood isolate. The patient was taken to the operating room where copious amounts of purulent material were drained and the forearm irrigated. All the muscles were intact, with no evidence of necrotizing fasciitis. A transthoracic echocardiogram showed no abnormalities. Postoperatively, the patient received a total of four weeks of intravenous and two weeks oral antimicrobial therapy, and made a slow but steady recovery. Six months after surgery, he had mild limitation of pronation and supination with reduced hand dexterity.

\footnotetext{
${ }^{1}$ Division of Infectious Diseases, ${ }^{2}$ Division of Orthopedic Surgery and the ${ }^{3}$ Department of Radiology, University of Alberta, Edmonton, Alberta Correspondence and reprints: Dr L Miedzinski, Division of Infectious Diseases, WMC 2E4.11, University of Alberta, 8440 - 112 Street, Edmonton, Alberta T6G 2B7. Telephone 780-407-7313, fax 780-407-7137, e-mail lil.miednzinski@ualberta.ca
} 


\section{DISCUSSION}

Despite advances in the diagnosis and therapy of bacterial disease, $S$ aureus remains a major cause of soft tissue infection. These infections can be extremely serious, particularly if associated with bacteremia, where the overall mortality has remained unchanged at $11 \%$ to $43 \%$ over the past 15 years (1). Diabetic patients, both insulin and noninsulin dependent, appear to have a higher incidence of severe and unusual infections due to this organism (2), as is seen in the present case. Postulated mechanisms for the latter include increased colonization rates, abnormal polymorphonuclear leukocyte function due to hyperglycemia, complement dysfunction, and both macro- and microvascular changes (3).

This case also demonstrates the difficulty in classifying soft tissue infections because their extent may only be ascertained reliably by surgical exploration (4). Although our case clearly was a deep space infection, it is less clear whether it was a variant of pyomyositis or a non-necrotizing fasciitis. Walling and Kaelin (5) described 'non-tropical' pyomyositis occurring in temperate countries and with an apparent predilection for patients with diabetes mellitus, where $56 \%$ of the infections were caused by $S$ aureus. The pathophysiology of this entity may involve the hematogenous seeding of a traumatic intramuscular hematoma (6), with initial pain and mild swelling followed by 'woody' induration of the area. A second phase with systemic symptoms occurs as isolated pockets of purulent material develop and a third, septic phase occurs when entire muscle groups are replaced by pus. This case may have been an early phase of pyomyositis or a noninvasive form of fasciitis with spread along fascial planes. In cases of deep space infections of the extremities where the exact location of the pathological process is unclear, a CT scan of the area may be a useful radiological modality. It may also aid in directing a surgical approach.

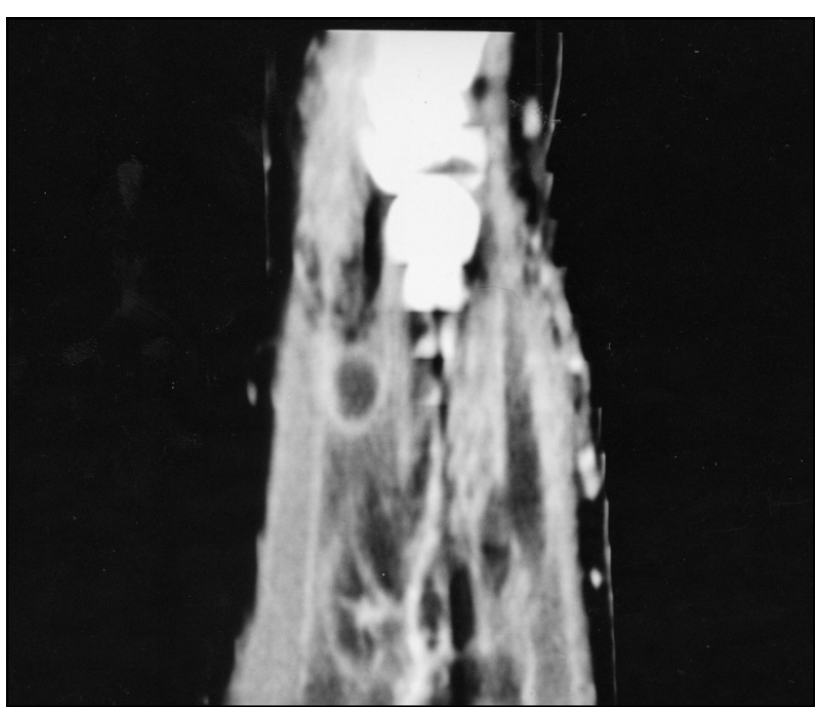

Figure 1) Enhanced sagittal image of the right forearm, showing multiloculated low attenuation in the interosseous space (with peripheral enhancement), extending both dorsally and ventrally

\section{REFERENCES}

1. Lowy, FD. Staphyloccus aureus infections. N Engl J Med 1998;339:520-32.

2. Breen JD, Karchmen AW. Staphylococcus aureus infections in diabetic patients. Infect Dis Clin North Am 1995;9:110-24.

3. McMahoon MM, Bistrian BR. Host defenses and susceptibility to infection in patients with diabetes mellitus. Infect Dis Clin North Am 1995;9:1-9.

4. Swartz MN. Cellulitis and subcutaneous tissue infections. Mandell, Douglas, and Bennett's Principles and Practice of Infectious Diseases, 4th edn. New York: Churchill Livingstone, 1995:909-29.

5. Walling DM, Kaelin WG. Pyomyositis in patients with diabetes mellitus. Rev Infect Dis 1991;13:797-802.

6. Sentochnik DE. Deep soft tissue infections in diabetic patients. Infect Dis Clin North Am 1995:9:53-64. 


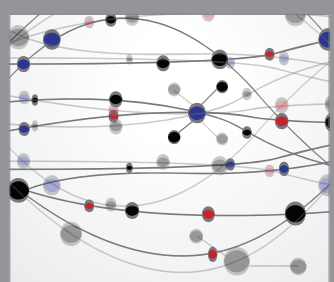

The Scientific World Journal
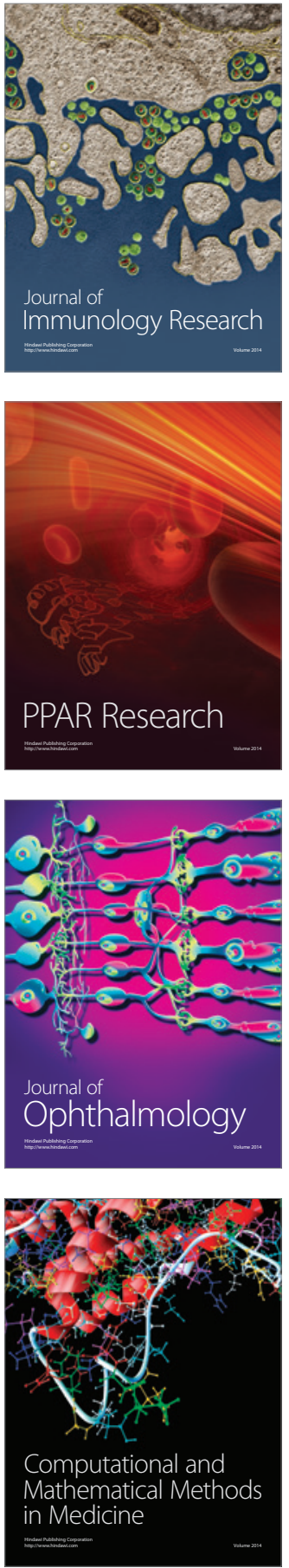

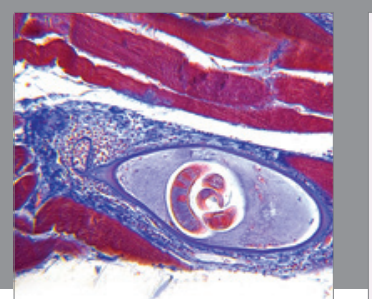

Gastroenterology Research and Practice

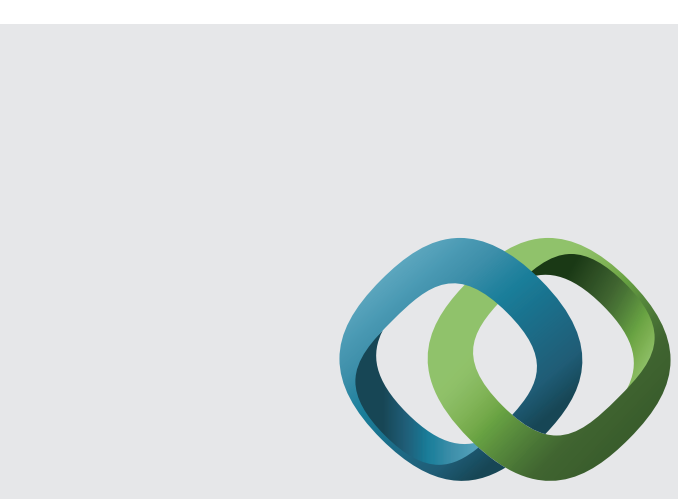

\section{Hindawi}

Submit your manuscripts at

http://www.hindawi.com
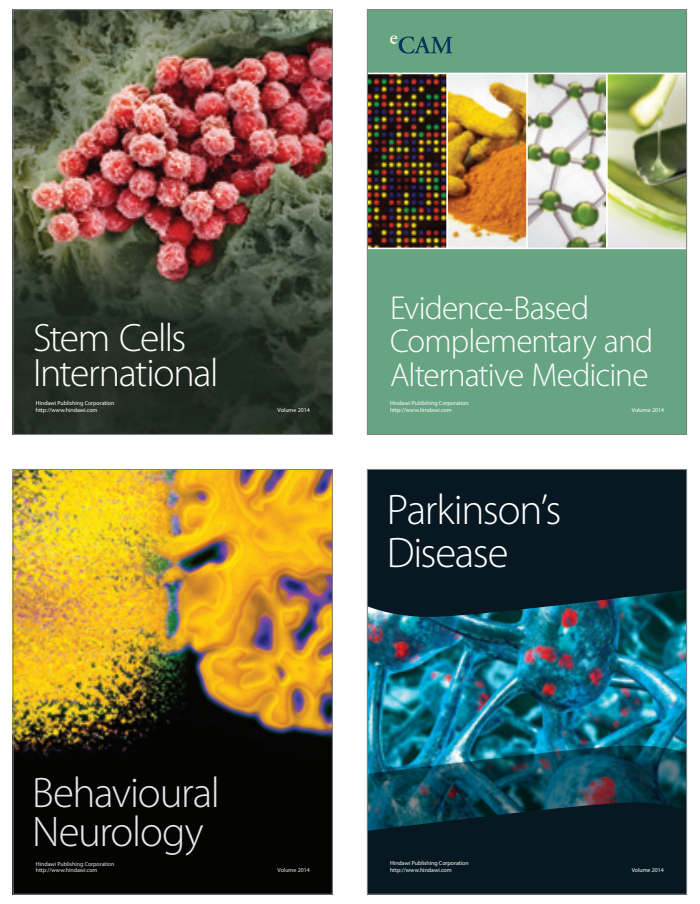
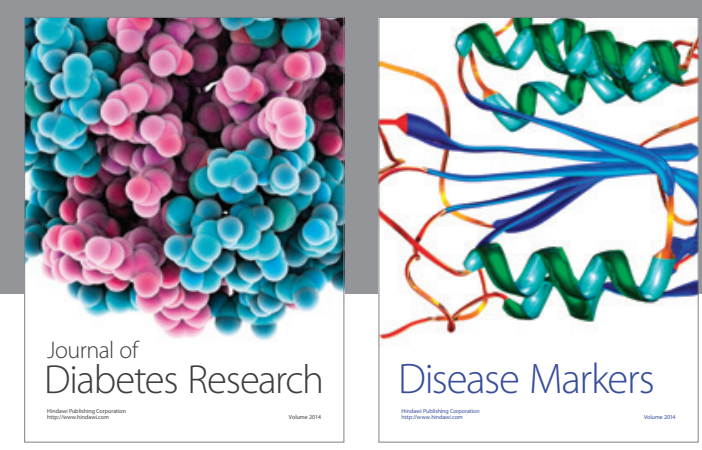

Disease Markers
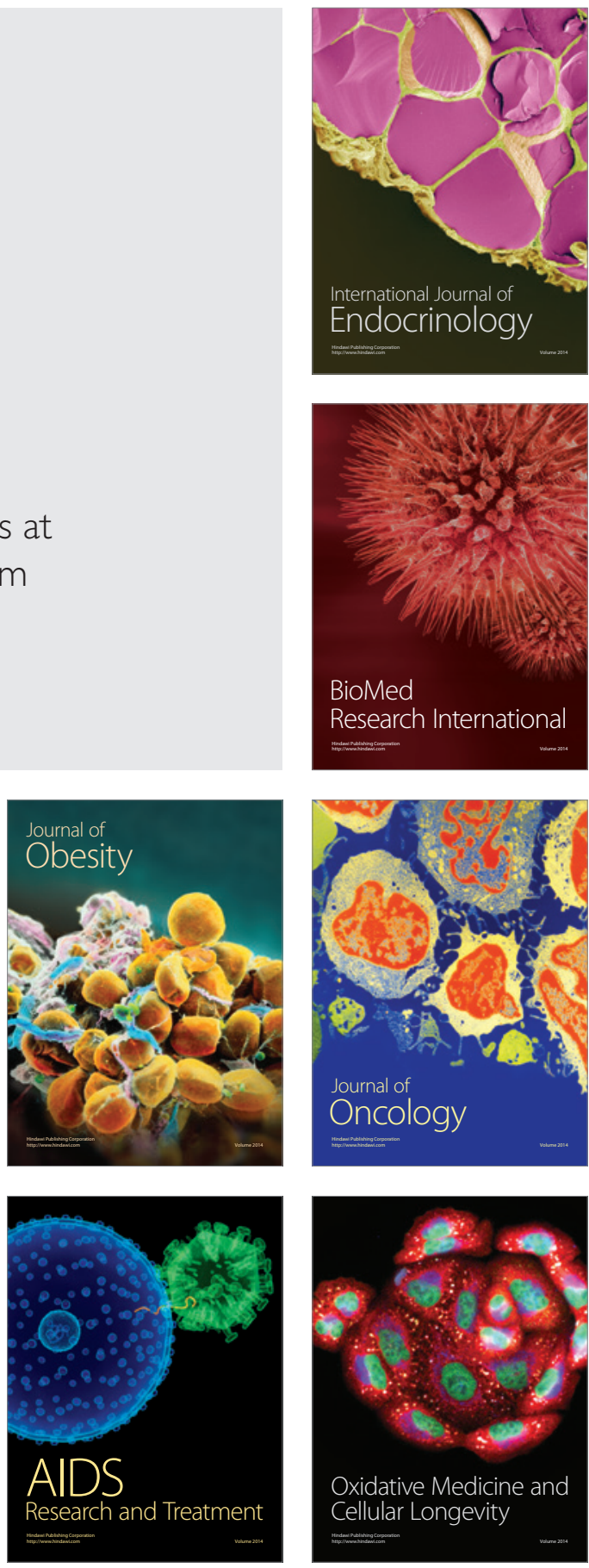